\title{
Principios éticos de los editores en las revistas científicas españolas de Comunicación, Educación y Psicología
}

\author{
Ethical principles of publishers in the Spanish scientific journals on Communication, Education and Psychology
}

\author{
Begoña GutiérRez SAn Miguel (1), Victoria Tur-Viñes (2), María Carmen FonseCA-Mora (3)
}

(1) Universidad de Salamanca, Facultad de Ciencias Sociales. Campus Miguel de Unamuno, 37007 Salamanca, España, bgsm@usal.es. (2) Universidad de Alicante, Campus de S. Vicente del Raspeig, Edif. de Ciencias Sociales, Ap. 99. 03080 Alicante, España, victoria.tur@ua.es (3) Universidad de Huelva, Facultad de Humanidades, Avda Tres de Marzo, s/n, Campus El Carmen, 21071 Huelva, España, fonseca@uhu.es

\begin{abstract}
Resumen
La responsabilidad ética de los editores de las revistas científicas se alza como asunto capital que, sobre todo, se hace visible en los casos de publicaciones fraudulentas. Se presenta un estudio exploratorio sobre la opinión que los editores de revistas científicas españolas de Comunicación, Educación y Psicología tienen sobre estas cuestiones. Se aplicó una metodología cuantitativa con un cuestionario electrónico enviado a 203 editores, compuesto de 19 items. Se observa que los editores dan una importancia esencial al rigor de la tarea de revisión, sustentado por pares ciegos. La mayoría de editores se consideran autodidactas y plantean la necesidad de profesionalizar el proceso editorial.
\end{abstract}

Palabras clave: Revistas científicas. Responsabilidad editorial. Códigos éticos. Comunicación. Educación. Psicología.

\section{Introducción}

Los editores de las revistas científicas tienen como objetivo fundamental la divulgación de los resultados de la actividad científica de los investigadores. Con el aumento de las publicaciones, surge la necesidad de establecer un arbitrio de funcionamiento ético internacional, en donde la responsabilidad recaiga fundamentalmente sobre los editores, cuestiones ya planteadas por Rodman en los años 70 del siglo pasado (1970).

La ética, como principio o pauta de la conducta vinculada al aspecto moral de las investigaciones, parece preocupar a los editores de las revistas científicas que se encuentran en la necesidad de dar un impulso a las mismas si pretenden tener una proyección en las bases de datos y en las redes de medición de la calidad y el impacto nacionales e internacionales, de forma que, los autores busquen publicar en ellas.

En los últimos años, la literatura sobre la práctica ética en la publicación científica ha proliferado

\begin{abstract}
The ethical responsibility of the editors of scientific journals is a cardinal issue, particularly following a series of fraudulent publications. This article presents the results of an exploratory study on the opinion of editors of Spanish scientific journals in the fields of Communication, Education and Psychology on these matters. The design of this research stems from a quantitative methodology and uses an electronic questionnaire that was submitted to 203 editors and had 19 items. The editors attach a great importance to the rigor of the reviewing process, carried out by blind peers. Most of the editors label themselves as autodidacts and raise the need to professionalize the editorial process.
\end{abstract}

Keywords: Scientific journals. Editorial responsibility. Ethical codes. Communication. Education. Psychology.

significativamente. El tema de los principios éticos de los editores de revistas científicas experimenta un interés creciente pareciendo recibir mayor atención en el contexto de las revistas de Ciencias Biomédicas (Rodman, 1970; Yankauer, 1990; Wilkes y Kravitz, 1995; Ray, 2002; Lindenauer, Benjamin, Naglieri Prescod, Fitzgerald y Pekow, 2002; Freda y Kearney, 2005; Marusic, Katavic y Marusic, 2007; Angelski, Fernández, Weijer y Gao, 2012; Bosch, Hernández, Pericas, Doti y Marušić 2012), que en el de las Ciencias Sociales -en adelante, CC. SS.- (Einhorn, 1971; McGinty, Roediger, 1987; Noble, 1989; Laband y Piette, 1994; Anderson, 1997; McGinty, 1999; Flowerdew, 2001; Hyde, 2001; Resnik, Patrone y Peddada, 2010; Baladrón Pazos y Correyero Ruiz, 2012; Tur, Fonseca y Gutiérrez, 2012). Los editores consideran la especificación y visibilidad de la normativa ética como modos de prevenir los conflictos derivados de su incumplimiento (Hunter, 2000; Wager, Fiack, Graf, Robinson y Rowlands, 2009; Resnik y otros, 2010; Bosch y otros, 2012; Angelski y otros, 2012). 
Muchas de las revistas de Ciencias Sociales españolas (CC. SS.) están mostrando signos de maduración viéndose en aumento el número de revistas españolas incluidas en las bases de datos consideradas de prestigio (Moreno-Pulido, López-González, Rubio-Garay, Saúl, SánchezElvira-Paniagua, 2013); bases de datos como Social Sciences Citation Index, Scopus o ERIH Plus, por citar algunas. Este hecho está provocando que los editores lleven a cabo un nuevo esfuerzo de implementación en sus publicaciones, fundamentando los códigos éticos como cuestión prioritaria.

Por otra parte, el caso Sokal (1998), así nombrado como distinción al físico que pergeñó la investigación con la intención de demostrar que una revista de humanidades la publicaría, como así ocurrió - Social Text-, (Sánchez Bonell, 2011), reactiva la necesidad de un rigor en los procesos de revisión en las revistas. La búsqueda de una ética editorial se fusiona con el proceso de calidad de las revistas como medio para mejorar y validar los textos científicos que se publican.

La responsabilidad ética de los editores cobra importancia a raíz de casos de fraude como el denunciado por la revista Science en el año 2013 "sobre un manuscrito con investigación totalmente falsa describiendo un fármaco milagroso, que se envió a 304 revistas y que fue aceptado en más de la mitad de las mismas", como comentan Baiget y Torres Salina (2013, p. 2).

En los últimos años y como reflejo del interés y la preocupación mostrados en el ámbito científico internacional, ha ido apareciendo en España literatura en la que se explicita la inquietud de los editores por las cuestiones éticas y en concreto en el ámbito de las CC. SS., en donde se manifiesta la necesidad de incorporar los modos y maneras de arbitrar el debate científico con normativas para los autores, para los evaluadores, para los editores, como Giménez y Alcaín (2006), Delgado López Cózar (2009), Fernández Quijada (2010), Castillo Esparcia y Carretón (2010), Santonja (2011), De Pablos (2011), Piedra (2012), o Tur-Viñes, López Sánchez, García del Castillo, López Orneales, Monstserrat Gauchi y Quiles Soler (2014), al igual que Bravo Toledo (2010), Castillo Esparcia, Rubio Moraga y Almansa Martínez (2013) o Tur-Viñes, Fonseca-Mora y Gutiérrez San Miguel (2012), entre otros.

\section{Preguntas de investigación}

Las preguntas de las que parte esta investigación giraron en torno a las siguientes cuestiones:
1. La percepción que el editor tiene sobre su responsabilidad ética en el proceso editorial.

2. Qué evidencias encontramos sobre la responsabilidad ética en la gestión editorial.

3. El tratamiento que tienen las indicaciones éticas en la revista.

4. Y finalmente si los editores están adheridos a protocolos éticos internacionales o tienen el suyo propio.

\section{Metodología}

\subsection{Objetivos y procedimiento}

El estudio realizado es de carácter exploratorio, siendo una primera aproximación con el deseo de cubrir otros campos en futuras investigaciones. Para ello se aplicó una encuesta compuesta por 19 items, a los editores de las revistas científicas españolas del campo de la Comunicación, de la Educación y la Psicología, sobre el tratamiento y la percepción de los principios éticos que observaban en ellas, con el objetivo de profundizar en la citada percepción de los editores de las 203 revistas científicas estudiadas. Para ello se trabajó con respuestas formadas por variables alfanuméricas, transformándolas a numéricas para obtener datos cuantitativos, previos al tratamiento estadístico, de forma que el entendimiento de los mismos y la definición de las variables diesen resultados claros y precisos. La metodología utilizada ha sido, por tanto, de índole cuantitativa.

Las revistas objeto de estudio fueron seleccionadas a partir del cruce de datos de los listados de 2012 de IN-RECS, Dialnet, Miar y RESH, teniendo en cuenta que dispusieran de un correo electrónico activo y estuvieran clasificadas dentro de los campos científicos contemplados en el estudio.

A continuación, se envió un cuestionario en línea en el que, se plantearon una serie de variables, de los cuales se han utilizado 19 ítems para llevar a cabo el actual artículo agrupadas en varios sectores:

1. Autoconciencia del editor sobre su responsabilidad: Utilizando una escala Likert de cuatro puntos (nada, poco, bastante, mucho) se quiso conocer la opinión de los editores sobre la responsabilidad ética de su cargo, en concreto, si ésta estaba configurada por los siguientes aspectos: confidencialidad, competencia/capacidad, imparcialidad e integridad, divulgación de conflicto de intereses y respeto de los plazos de revisión. 
2. Prácticas editoriales responsables y grado de transparencia: Se preguntó si los editores observaban determinadas prácticas editoriales responsables y hasta qué punto las mismas eran transparentes.

3. Responsabilidad en las interacciones editorautor en relación a los códigos éticos: Con una escala Likert de cuatro puntos (nunca, a veces, con frecuencia, siempre) se indagó la existencia de un registro auditable o verificable de las interacciones editor-autor-evaluadores, si las decisiones editoriales se comunican de forma motivada, y si se acusa recibo de todas las interacciones.

4. Ubicación de las indicaciones éticas: Se preguntó si existían indicaciones éticas en las normas a autores o revisores, si constan indicaciones éticas publicadas para el comité de redacción o editorial y la existencia de un apartado propio y público que unifique todas las indicaciones éticas.

5. Adhesión a protocolos éticos de organizaciones reconocidas: Committee on Publication Ethics (Cope), Council of Science Editors (CSE), International Committee of Medical Journal Editors (Icmje), Consejo Superior de Investigaciones Científicas (Csic), World Association of Medical Editors (Wame), o si cuentan con un protocolo propio.

6. Necesidades de asesoramiento o formación de los editores en temas éticos.

La herramienta de medida utilizada fue un cuestionario semi-estructurado, planteado siguiendo las recomendaciones de Dillman (2000) para el diseño de preguntas, haciendo una adaptación al formato en línea.

El cuestionario fue remitido en dos ocasiones consecutivas durante el año 2013, obteniéndose del total de las 203 revistas, 81 contestaciones, lo que refleja una tasa de respuesta del $39,9 \%$. Los resultados obtenidos pueden parecer válidos al establecer una comparación con estudios semejantes en donde se observaron resultados del $44 \%$ en Wager y otros (2009), el $32,5 \%$ en Tavares de Matos Cardoso (2011) o el 33\% en Angelski y otros (2012).

Para la validación de la muestra y su sistematización en función de la obtención de resultados, se utilizó el programa estadístico para análisis predictivo de resultados fiables SPSS (SPSS 21).

\section{Resultados}

Las respuestas obtenidas de los editores de las revistas objeto de estudio, revelan que existen indicaciones éticas incluidas en las normas para los autores, los revisores o para el equipo editorial de forma mayoritaria (77\%). En el $38 \%$ de los casos dichas indicaciones se dirigen a autores, en el $24 \%$ a revisores y en el $15 \%$ al equipo editorial.

4.1. En relación con la autoconciencia del editor sobre los principios éticos más significativos que observan en sus revistas

La libertad de expresión de los autores se tiene en cuenta, ya sea implícita o explícitamente, en la mayoría de los casos con un porcentaje elevado y significativo $(99 \%)$. Curiosamente existe alguna revista de la muestra seleccionada para el estudio que no contempla este concepto $(1 \%)$.

\begin{tabular}{lccc}
\hline Principio ético & Expl. & Impl. & No \\
\hline $\begin{array}{l}\text { La libertad de expresión } \\
\text { de los autores se tiene en cuenta }\end{array}$ & $45 \%$ & $54 \%$ & $1 \%$ \\
\hline $\begin{array}{l}\text { La atención a la diversidad } \\
\text { y minorias se tiene en cuenta }\end{array}$ & $17 \%$ & $65 \%$ & $18 \%$ \\
\hline $\begin{array}{l}\text { El proceso de reclamación } \\
\text { se tiene en cuenta }\end{array}$ & $28 \%$ & $65 \%$ & $7 \%$ \\
\hline $\begin{array}{l}\text { La libertad de decisión de los } \\
\text { evaluadores se tiene en cuenta }\end{array}$ & $64 \%$ & $35 \%$ & $1 \%$ \\
\hline $\begin{array}{l}\text { La libertad de decisión del editor } \\
\text { se tiene en cuenta }\end{array}$ & $41 \%$ & $49 \%$ & $10 \%$ \\
\hline $\begin{array}{l}\text { Se reconoce la responsabilidad } \\
\text { del editor en la preservación } \\
\text { del anonimato de la revisión }\end{array}$ & $74 \%$ & $22 \%$ & $4 \%$ \\
\hline $\begin{array}{l}\text { La confidencialidad de editores } \\
\text { y revisores se tiene en cuenta }\end{array}$ & $73 \%$ & $26 \%$ & $1 \%$ \\
\hline $\begin{array}{l}\text { Se desaconsejan las prácticas } \\
\text { de manipulación de citas }\end{array}$ & $57 \%$ & $30 \%$ & $13 \%$ \\
\hline $\begin{array}{l}\text { La declaración de derechos } \\
\text { de autor se tiene en cuenta }\end{array}$ & $69 \%$ & $27 \%$ & $4 \%$ \\
\hline $\begin{array}{l}\text { Existen indicaciones sobre } \\
\text { coautoría }\end{array}$ & & & \\
\hline $\begin{array}{l}\text { Existen indicaciones } \\
\text { sobre la responsabilidad moral } \\
\text { y ética de los autores }\end{array}$ & $29 \%$ & $50 \%$ & $21 \%$ \\
\hline $\begin{array}{l}\text { Se recaba el compromiso } \\
\text { del autor con las directrices } \\
\text { internacionalmente aceptadas } \\
\text { cuando la investigación implique } \\
\text { a personas o animales }\end{array}$ & & & \\
\hline & & & \\
\hline
\end{tabular}

Tabla I. Autoconciencia del editor sobre los principios éticos observados en sus revistas

La atención a la diversidad y a las minorías, tanto culturales como sexuales, se tiene en cuenta en un porcentaje de un $82 \%$ frente a un $18 \%$ que no lo hace. El proceso de reclamación está registrado en la mayoría de las revistas con un porcentaje del $93 \%$. Un $7 \%$ no lo contempla. La libertad de decisión de los evaluadores se mantiene en el 99\%. La libertad de decisión es un asunto prioritario en el $90 \%$, frente al $10 \%$ que dice consensuar sus decisiones con el resto del consejo. Igualmente, un $96 \%$ de los editores re- 
conoce como responsabilidad suya la preservación del anonimato en el proceso de revisión. El compromiso de confidencialidad o discreción entre editores y revisores - de forma que ninguna de las partes pueda utilizar la información para sus propios fines- se da en un $99 \%$ de las revistas. Se desaconsejan las prácticas de manipulación de citas en un $87 \%$ y un $13 \%$ que no lo deja patente ni explícita ni tácitamente. La cesión de derechos de autor se tiene en cuenta en la casi totalidad de los casos, como un medio para amparar la ética editorial, salvo en un $4 \%$ de las respuestas de los editores. Las indicaciones para la coautoría aparecen en el $51 \%$ de los casos y el $49 \%$ no existe ninguna indicación. Las normas éticas para los autores están muy definidas en el $79 \%$ de las revistas. El $21 \%$ no tienen ninguna. En este apartado se plantean los dilemas morales casi a la par; el 55\% de las revistas incorporan normativa internacional en torno a la manipulación de animales o personas en las investigaciones y en consecuencia en los resultados de la investigación en sus revistas, frente a un $45 \%$ que no lo hace.

\subsection{Prácticas editoriales responsables} y grado de transparencia

En este apartado destacan de forma significativa las siguientes variables (Tabla II).

\begin{tabular}{lrccc}
\hline Principio ético estudiado & Nunca & A veces & Frec. & Siemp \\
\hline $\begin{array}{l}\text { Existe un registro auditable } \\
\text { de las interacciones }\end{array}$ & $21 \%$ & $20 \%$ & $12 \%$ & $47 \%$ \\
\hline $\begin{array}{l}\text { Las decisiones editoriales } \\
\text { se comunican de forma } \\
\text { motivada }\end{array}$ & $1 \%$ & $5 \%$ & $10 \%$ & $84 \%$ \\
\hline $\begin{array}{l}\text { Se acusa recibo de todas } \\
\text { las interacciones }\end{array}$ & $4 \%$ & $3 \%$ & $8 \%$ & $85 \%$ \\
\hline $\begin{array}{l}\text { La calidad de los textos se } \\
\text { fundamenta en la revisión } \\
\text { ciega por pares expertos }\end{array}$ & $2 \%$ & $1 \%$ & $2 \%$ & $95 \%$ \\
\hline
\end{tabular}

Tabla II. Prácticas editoriales responsables y grado de transparencia

Los datos revelan que los editores de algo más de la mitad de las revistas objeto de estudio (52\%) manifiestan que nunca, a veces o con frecuencia tienen constancia de un registro auditable o verificable, frente a los que afirman tenerlo (47\%). Las decisiones editoriales se comunican de forma motivada en un porcentaje mayoritario (84\%), frente a las que lo hacen a veces, con escasa o ninguna frecuencia (16\%). Por el contrario, acusan recibo de todas las interacciones (85\%) un número mayoritario de revistas frente a un porcentaje muy inferior (15\%) en las que apenas o casi nunca se hace.
El proceso de evaluación es llevado a cabo, de forma mayoritaria, por una revisión de pares ciegos de expertos (95\%), como medio de fundamentar la calidad ética de los textos científicos que se publican en las revistas objeto de estudio. Solo en un número pequeño no lo hacen siempre $(5 \%)$.

\subsection{Responsabilidad en las interacciones} editor-autor en relación a los códigos éticos

La responsabilidad ética de las revistas científicas en el campo de las CC. SS. de las revistas objeto de estudio se fundamenta en diversas cuestiones (Tabla III). El proceso editorial se rige por el concepto de confidencialidad como cuestión capital en un $67 \%$ de los casos, frente a un $2 \%$ que considera no ser uno de los factores más importantes. La competencia o capacidad del editor y en consecuencia de la revista se valora de forma bastante elevada en el $94 \%$ de los casos frente al $6 \%$ que no lo ve así. La imparcialidad e integridad se contempla como una de las normas éticas más importantes por la mayoría de los editores con un $96 \%$ de los casos frente a un $4 \%$ que no lo considera así. El de conflicto de intereses se valora en un $67 \%$ mucho o bastante, frente a un $33 \%$ que lo valora poco o nada. El respeto a los plazos para completar la revisión se ve como una responsabilidad ética del editor en $78 \%$ de los casos frente al $22 \%$ que lo contempla como una responsabilidad ajena al mismo.

\begin{tabular}{lcccc}
\hline Variables & Nada & Poco & Bastante & Mucho \\
\hline Confidencialidad & $2 \%$ & $9 \%$ & $22 \%$ & $67 \%$ \\
\hline $\begin{array}{l}\text { Competencia / } \\
\text { Capacidad }\end{array}$ & $0 \%$ & $6 \%$ & $27 \%$ & $67 \%$ \\
\hline $\begin{array}{l}\text { Imparcialidad / } \\
\text { Integridad }\end{array}$ & $0 \%$ & $4 \%$ & $18 \%$ & $78 \%$ \\
\hline $\begin{array}{l}\text { La divulgación } \\
\text { del conflicto de } \\
\text { intereses }\end{array}$ & $9 \%$ & $24 \%$ & $32 \%$ & $35 \%$ \\
\hline $\begin{array}{l}\text { El respeto a los plazos } \\
\text { para completar la } \\
\text { revisión }\end{array}$ & $4 \%$ & $18 \%$ & $49 \%$ & $29 \%$ \\
\hline
\end{tabular}

Tabla III. Opinión de los editores sobre aspectos integrantes de la responsabilidad ética

\subsection{Adhesión a protocolos éticos de organizaciones reconocidas}

El $44 \%$ de las revistas no tienen reflejados códigos éticos. Las que tienen normas éticas de elaboración propia suponen un $32 \%$ del total de las revistas encuestadas. El 15\% están asociadas a las normas planteadas por el CSIC (Centro Superior de Investigaciones Científicas). El 2\% se rige por los planteamientos éticos del Cope (Committee on Publication Ethics), el $1 \%$ por las 
del CSE (Council of Science Editors). En el 6\% de ellas dicen tener normas éticas correspondientes a otros campos que no especifican.

\section{Discusión de resultados}

Si bien el estudio llevado a cabo incluye un porcentaje significativo de revistas científicas publicadas en la red (no podemos afirmar que sólo sean en línea) dentro de los campos de la Comunicación, Educación y Psicología españoles, sus datos no pueden extrapolarse al resto de las revistas del campo de las Ciencias Sociales por el carácter exploratorio de esta primera investigación. Quedan por analizar el resto de campos científicos (Antropología, Documentación, Ciencia Política, Economía, Geografía y Urbanismo).

La tasa de respuesta obtenida es contemplada de forma global, sin hacer separación entre las áreas dado que el campo científico en el que están clasificadas las revistas por los propios editores no está delimitado de forma absoluta perteneciendo a varios campos (Educación y Comunicación, por ejemplo) por lo que entorpece la extracción de conclusiones diferenciales definitivas. Este hecho se pude indicar como una limitación del estudio.

Varios son los ejes investigadores de este artículo; el primero está relacionado con la existencia de normativa ética en las revistas objeto de estudio, el segundo con los principios éticos más relevantes destacados por los responsables de las citadas revistas, el tercero sería el tratamiento de los principios éticos y el cuarto la responsabilidad que sienten los editores de las citadas revistas.

En relación con la primera cuestión que plantea el estudio existencia o al menos presencia de los principios éticos en las revistas objeto de estudio. El resultado confirma que un porcentaje pequeño de ellas (11\%) tiene especificadas las normas éticas en un apartado especial, propio y público. En el resto de las revistas, en un porcentaje mayoritario $(77 \%)$, las indicaciones éticas aparecen en apartados dirigidos a autores, revisores o equipo editorial, entremezcladas con otras informaciones.

La normativa ética de alguna de las revistas objeto de estudio (18\%) se rige por las líneas planteadas en los grandes comités de ética internacional - sello Cope o CSE-, provenientes del ámbito de la biomedicina o por las planteadas por el Centro Superior de Investigaciones Científicas Español (15\%). La normativa ética propia supone un porcentaje elevado en la muestra objeto de estudio (32\%), frente a un número mayoritario que no parece necesitar la presencia de dichas cuestiones en sus revistas (44\%). Se evidencia cierta necesidad de homogeneización o estandarización en las normativas propias.

Los principios éticos más significativos encontrados en las revistas científicas objeto de estudio dan una prioridad mayoritaria a tres cuestiones: la libertad de expresión de los autores, la libertad de decisión de los evaluadores y la confidencialidad entre revisores y editores (99\%). Un dato curioso a destacar es el porcentaje del $1 \%$ que no contempla la libertad de expresión en sus cuestiones éticas.

A continuación, ocuparían el siguiente escalafón de importancia la declaración de los derechos de autor y la preservación del anonimato de la revisión de los artículos como una cuestión ligada al editor $(96 \%)$ y el proceso de reclamación $(93 \%)$, contemplando que la libertad de decisión del editor es decisiva en una mayoría de las revistas objeto de estudio (90\%). La cuestión relacionada con el anonimato queda reflejada en el estudio planteado por Coslado et al. (2011), en el que constataron que la mayoría de las revistas científicas españolas respetaban este código de confidencialidad $(94 \%)$.

En el siguiente orden de importancia se coloca la manipulación de citas (87\%), como un problema importante que desaconseja esta mala praxis. Al respecto, Valero Matas, Jiménez García y Coca $(2013,10)$ comentan en el estudio llevado a cabo para analizar la evaluación de trabajos científicos en revistas:

Es sabido que nuestro modelo tiene unos vicios motivados por la llamada endogamia académica, la cual ha dado lugar a la silicosis burocrática, que hace de la valoración cuantitativa un mero pasaje institucional, sin dotarlo del verdadero sentido del mismo.

En él añaden que el incumplimiento de las normativas éticas se da fundamentalmente en torno a ciertos parámetros como son las citas recíprocas o las autocitas sin justificar.

Finalmente, se comprueba que los editores y las revistas científicas son sensibles a la diversidad social y a las minorías (82\%). La normativa ética presenta una mención clara y específica cuando se refiere a los autores, siendo las indicaciones sobre la responsabilidad moral o ética las que se destacan en este apartado mayoritario $(79 \%)$. Resulta curioso observar que existe un porcentaje de revistas que no considera importantes estas cuestiones $(21 \%)$.

Destaca que la mitad de los principios éticos estudiados están considerados de forma implícita en el proceso editorial con más frecuencia y no se comuniquen de forma pública, explícita y 
clara. Ello indica que la transparencia e información ética todavía tiene un tratamiento desordenado y mejorable en las revistas investigadas.

Uno de los aspectos más acuciantes son las indicaciones sobre coautoría, inexistentes en casi la mitad de las revistas, a pesar de que suponen un indicador de calidad para la inclusión en algunas bases de datos. En segundo lugar, urge recabar el compromiso del autor con las directrices internacionalmente aceptadas cuando la investigación implique a personas. Es una práctica habitual en Ciencias de la Salud, pero el objeto de las Ciencias Sociales es la persona o el grupo social y también existen directrices a observar en este ámbito. Uno de los que tiene mayor recorrido es el código internacional ICC/ESOMAR para la práctica de la investigación social y de mercados que data de 1948, cuya última actualización se produjo en 1994. Estas cuestiones se detallan minuciosamente en una publicación de Wiley (2014), sobre buenas prácticas en las publicaciones científicas. El porcentaje de revistas que tienen indicaciones éticas relacionadas con el trabajo con seres humanos alcanza el $55 \%$ frente al $45 \%$ que no dispone de ellas.

La relación ética entre editores y autores se establece esencialmente en la comunicación de la evaluación, el registro auditable de las acciones, el informe de las decisiones editoriales y en el envío de las motivaciones presentadas por los evaluadores.

Los evaluadores constituyen el apoyo esencial de los editores, recurriendo a la evaluación por pares ciegos de expertos en la materia; "eso otorga al editor el carácter de gatekeeper, de guardador de accesos, de garante de la legalidad y la legitimidad de los productos", comenta Lolas (2002, p. 228). Este es el modelo adoptado por la mayoría de las revistas objeto de estudio en un porcentaje casi absoluto (95\%). De forma semejante, Coslado et al. (2011) verifican que existe un arbitraje de este tipo en un número elevado de revistas (85\%). A pesar de ello, diversas voces se alzan para cuestionar la validez o calidad de este proceso. Así, Valero Matas el al. (2012, p. 13) reclaman que se evalúen las cuestiones cualitativas

La revisión por pares de los proyectos y trabajos de investigación en los términos actuales es insuficiente para concederle a una revista el grado de calidad.

Igualmente, Baladrón Pazos y Correyero Ruiz (2012, p. 40) señalan que "las respuestas de los expertos evidencian que los procesos no son siempre suficientemente rigurosos".
Otros aspectos relevantes para mayoría de los editores son el envío de las decisiones motivadas a los autores (84\%) y el remitir acuse de recibo a los mismos $(79 \%)$, guardando constancia en un registro auditable $(52 \%)$, frente a casi la otra mitad que no lo consideran necesario. Este hecho parece reflejar, en cierta medida, la inexperiencia en la gestión de las revistas o la falta de formación de los editores, cuestión apuntada por ellos mismos. "Las revistas deben considerar el envío de copias de toda la correspondencia a todos los autores que firman un artículo", comenta Wiley (2014).

La responsabilidad ética de los editores parece ser considerada el motor principal en el funcionamiento de las revistas. Una de las prioridades, como ya se ha apuntado es la imparcialidad e integridad $(96 \%)$, al igual que la competencia o capacidad del editor (94\%). El respeto a los plazos para completar la revisión se ve como una responsabilidad ética del editor en un porcentaje menor $(78 \%)$, achacando al compromiso directo y personal de los propios revisores (22\%). El caso mostrado por García Puig et al. (2010) sobre el papel que han desempeñado los revisores de la Revista Clínica Española, refleja porcentajes semejantes a los que aquí se obtienen (75\%), coincidiendo en que los revisores han cumplido con los plazos establecidos por los editores de la revista, progresión que se ha ido estabilizando en los estudios que realizaron en los años posteriores hasta el 2014. La comparación es significativa, dado que los resultados obtenidos en su estudio reflejan la importancia y rol que otorgan a los revisores, puesto que los propios autores del informe son los editores de la revista.

La confidencialidad y la divulgación de conflicto de intereses se sitúan en un mismo porcentaje de importancia (67\%). En el caso de la confidencialidad este porcentaje corresponde a los que les asignan mucha importancia, mientras que en el del conflicto de intereses se combinan las respuestas de los que lo consideran muy importante y bastante importante. Los revisores pueden ser de gran utilidad para alertar a los editores en la detección de conflicto de intereses igual que se mantiene el respeto por la confidencialidad por parte del editor de esta detección, cuestión destacada por la guía Wiley (2014).

Se puede afirmar que los editores declaran en porcentajes elevados ser autodidactas (98,8\%) -Tur-Viñes, Fonseca-Mora, Gutiérrez San Miguel (2014) - y reclaman un replanteamiento de la situación editorial tanto en cuanto a la especialización, como en algunos casos a la búsqueda de gestores expertos en la materia que oficien las revistas: "la profesionalización de la gestión im- 
plica la dotación de recursos humanos y económicos suficientes que garanticen la estabilidad, la calidad y el rigor en la edición de la revista" apuntan Rodríguez Yunta y Giménez Toledo (2013, 43). En el estudio llevado a cabo por Ray (2002), el $69 \%$ de los editores manifestaban la necesidad de algún tipo de formación editorial previa.

\section{Conclusiones}

Para finalizar podemos constatar que las preguntas de esta investigación en torno a la responsabilidad ética del editor, la gestión de las normas éticas, el reflejo en las normas de las mismas, al igual que la importancia de destacar los protocolos éticos seguidos por los editores de las revistas objeto de estudio se confirman como temas de preocupación para los editores de revistas científicas y tienen un tratamiento desigual, no consensuado y carente de estandarización.

Se ha evidenciado que la proliferación de revistas científicas, la necesidad de publicación por parte de los autores y la búsqueda de la calidad científica relacionada con las presencia e incidencia de normas éticas que amparen y protejan el proceso editorial configuran un escenario donde la necesidad de una práctica ética rigurosa adquiere cada vez más importancia a la hora de gestionar el proceso editorial.

Los resultados de la investigación revelan tanto la importancia como la relevancia de las consideraciones éticas de la publicación científica y apuntan los aspectos que todavía son susceptibles de atención y mejora en el incipiente cambio de paradigma que se vislumbra.

\section{Agradecimientos}

Estudio financiado por el Ministerio de Ciencia e Innovación de España, dentro del Plan Nacional de I+D+i (EDU201113034-E), acción complementaria: Observatorio de revistas científicas de ciencias sociales.

\section{Referencias}

Anderson, Paul (1997). Gatekeepers and the quality of the journal literature: Findings from a survey of journal editors into the issue of alleged excessive publication in scholarly and scientific journals. // Serials Review. 23:2, 45-57.

Angelski, Claudia; Fernández, Conrad; Weijer, Charles; Gao, Jiali (2012). The publication of ethically uncertain research: attitudes and practices of journal editors. // BCM medical ethics, 13:4.

Baiget, Tomás; Torres Salina, Daniel (2013). Informe sobre publicación en revistas científicas. // Informe APEI (Asociación Profesional de Especialistas en Información). 1- 95.

Baladrón Pazos, Antonio J.; Correyero Ruiz, Beatriz (2012). Futuro de las revistas científicas de comunicación en España. // El profesional de la información. 21:1, 34-42.

Bosch Xaxier, Hernández Cristina; Pericas, Juan Manuel; Doti, Pamela; Marušić, Ana (2012). Misconduct Policies in High-Impact Biomedical Journals. DOI:10.1371/ journal.pone.0051928.
Bravo Toledo, Rafael (2010). Aspectos éticos en las publicaciones científicas. http://www.infodoctor.org/rafabravo/ fraude.htm (2013-04-02).

Castillo Esparcia, Antonio; Carretón, María del Carmen (2010). Investigación en Comunicación. Estudio bibliométrico de las Revistas de Comunicación en España. // Revista Comunicación y Sociedad. 23:2, 289-327. http://www.unav.es/fcom/comunicacionysociedad/es/resumen.php?art_id=372 (2013-04-02).

Castillo Esparcia, Antonio; Rubio Moraga, Angel; Almansa Martínez, Ana (2012). La Investigación en comunicación: análisis bibliométrico de las revistas de mayor impacto del ISI. // Revista Latina de Comunicación Social. 67, 248-270.

Comité Internacional de Directores de Revistas Médicas (1999). Declaraciones del Comité Internacional de Directores de Revistas Médicas anexas a las normas de Vancouver. // Revista Medícal Hered 10: 3. 119-124.

Coslado, Ma Ángeles; Lacunza, Izascun; Ros, Germán (2011). Evaluación de la calidad de revistas científicas españolas: análisis de sus procesos de revisión. // El Profesional de la Información. 20:2,160-164.

Daza, Sandra; Arboleda, Tania (2007). Comunicación pública de la ciencia y la tecnología en Colombia: ¿políticas para la democratización del conocimiento?. // Signo y Pensamiento. 50:26, 101-125.

De Pablos, José Manuel (2011). Productividad científica e índice de impacto de las revistas españolas de Comunicación. // Cuadernos Artesanos de Latina. 10, 23-37. http://www.revistalatinacs.org/067/cuadernos/10 Fonseca_interior.pdf. (2013-04-02).

Delgado López Cózar, Emilio (2009). Las revistas españolas de Comunicación a través del IN-RECS/IN-RECJ (Índice de impacto de las revistas Españolas de Ciencias Sociales y Jurídicas). // Actas del I Congreso Internacional Latina de Comunicación, Tenerife.

Dillman, Don (2000). The Tailored Design Method. In Mail and Internet Surveys. 2nd ed. New York: John Wiley y Sons.

Einhorn, Hillel (1971). Responsibility of journal editors and referees. // American Psychologist 26:6. 600.

Elvira Paniagua, Ángeles (2013). Evolución de las revistas españolas de Ciencias Sociales en el Journal Citation Reports (2006-2010) y su valoración como indicio de calidad en la normativa evaluadora española. // Revista española de Documentación Científica. DOI: http://dx.doi.org/10.3989/redc.2013.3.987

Fonseca-Mora, $\mathrm{M}^{\mathrm{a}}$ Carmen; Tur-Viñes, Victoria; Gutiérrez San Miguel, Begoña (2014). Ética y revistas científicas españolas de Comunicación, Educación y Psicología: la percepción editora. // Revista española de Documentación Científica. http://dx.doi.org/10.3989/redc.2014.4.1151.

Fernández Quijada, David (2010). El perfil de las revistas españolas de comunicación (2007-2008). // Revista española de Documentación Científica. 33:4, 553-581. http://redc.revistas.csic.es/index.php/redc/article/view/671/746 (2013-04-02).

Flowerdew, Jhon (2001). Attitudes of journal editors to nonnative speaker contributions. // Tesol Quarterly. 35:1, 121-150.

Freda, Margaret; Kearney, Margaret (2005). An international survey of nurse editors' roles and practices. // Journal of Nursing Scholarship. 37:1, 87-94.

García Puig, Juan; Alonso Vega, Gaspar; Ríos Blanco, Juan José (2010). Informe de los editores, 2010. // Revista Clínica Española. 211:1, 36-45

García Puig, Juan; Alonso Vega, Gaspar; Ríos Blanco, Juan José (2011). Informe de los editores, 2010. // Revista Clínica Española. 212:1, 31-39 
García Puig, Juan; Alonso Vega, Gaspar; Ríos Blanco, Juan José (2013). Informe de los editores, 2010. // Revista Clínica Española. 214, 38-45

Giménez Toledo, Elea; Alcain Partearroyo, María Dolores (2006). Estudio de las revistas españolas de Periodismo. // Revista Comunicación y Sociedad. 19:2, 107- 131. http://www.unav.es/fcom/comunicacionysociedad/ es/articulo.php?art_id=62. (2013-04-02).

Gregorio, Orlando (2007). Análisis bibliométrico y de calidad de la revista Signo y Pensamiento (1982-2006). // Signo y Pensamiento 50: 26. 22-32.

Hyde, Janet Shibley (2001). Reporting effect sizes: The roles of editors, textbook authors, and publication manuals. Educational and Psychological Measurement.

Icc/esomar (1948, última actualización en 1994). Código internacional para la práctica de la investigación social y de mercados. http://www.autocontrol.es/pdfs/pdfs_co digos/cod-aneimo(ICC\%20ESOMAR).pdf. (2013-04-02).

Laband, Debie; Piette, Michael (1994). Favoritism versus search for good papers: Empirical evidence regarding the behavior of journal editors. // Journal of Political Economy. 194-203.

Lindenauer, Peter; Benjamin, Evan; Naglieri-Prescod, Debbie; Fitzgerald, J. Mark; Pekow, Penelope (2002). The role of the institutional review board in quality improvement: a survey of quality officers, institutional review board chairs, and journal editors. // The American journal of medicine. 113:7, 575-579.

Lolas Stepke, Fernando (2000). Ética de la publicación médica: legalidad y legitimidad. // Acta Bioethica. 6:2, 283291.

Mayta Tristán, Percy; Curioso, Walter (2009). Política editorial ante la detección de una publicación redundante. // Revista Peruana de Medicina Experimental y Salud Pública. 26:1.

Marusic, Ana; Katavic, Vedran; Marusic, Matko (2007). Role of editors and journals in detecting and preventing scientific misconduct: strengths, weaknesses, opportunities, and threats. // Medicine and law. 26:3, 545.

McGinty, Stephen (1999). Gatekeepers of knowledge: journal editors in the sciences and the social sciences. // Greenwood Publishing Group.

Noble, Kim (1989). Publish or perish: What 23 journal editors have to say. // Studies in Higher Education. 14:1, 97-102.

Olivera Betrán, Javier (2011). Tendencias en la edición y en la mejora de la calidad de las revistas científicas españolas de ciencias sociales. // Apunts. Educación física y deportes.104, 5-10.

Piedra, Yelina (2012). Campo científico de la Comunicación: examinando su estructura intelectual a través del análisis de cocitación. // Francisco Javier Herrero (coord.). Elegir dónde publicar. Transferencia de la investigación en el área de Comunicación. La Laguna (Tenerife). Sociedad Latina de Comunicación Social. 49-68.

Ray, Joel G. (2002). Judging the judges: the role of journal editors. // QJM. 95:12, 769-774.

Resnik, David; Patrone, Daniel; Peddada, Shyamal (2010). Research misconduct policies of social science journals and impact factor. // Accountability in research: policies and quality assurance. 17:2, 79-84.

Rodman, Hyman (1970). The moral responsibility of journal editors and referees. // The American Sociologist. 351-357.

Rodríguez Yunta, Luis; Giménez Toledo, Elea (2013). Fusión, coedición o reestructuración de revistas científicas en humanidades y ciencias sociales. // El profesional de la información. 22:1, 36-45

Roediger III, Henry L. (1987) The role of journal editors in the scientific process. // Jackson, Douglas N.; Rushton, J.
Philippe (Ed) (1987). Scientific excellence: Origins and assessment, (222-252). Thousand Oaks, CA, US: Sage Publications, Inc, 381.

Rojas Revoredo, Verónica; Huamani, Charles; Mayta Tristán, Percy. 2007. Plagio en publicaciones científicas en el pregrado: experiencias y recomendaciones. // Revista Médica de Chile. 135. 1087-1088.

Sánchez Bonell, David (2008). El asunto Sokal: un problema de actitud científica. // Educación Científica y Desarrollo Social. Bogotá. Colombia. 109-115.

Santana Álvarez, Jorge (2012). Ética, investigación y publicación. // Revista Archivo Médico de Camagüey.16:5.

Santonja, Lola (2011). Informe sobre calidad de las revistas en el área de Comunicación. Versión 2011. Revistas mejor valoradas en los sistemas de evaluación. Madrid: Biblioteca de Humanidades, Comunicación y Documentación. Universidad Carlos III.

Sokal, Alan D. (1998). Transgrediendo los límites: hacia una hermenéutica transformadora de la gravedad cuántica. Madrid: La Balsa de la Medusa. 45, 9-42.

Tabares de Matos Cardoso, María Manuela (2011). El peer review de las revistas científicas en Humanidades y Ciencias Sociales: políticas y prácticas editoriales declaradas. // Revista Española de Documentación Científica. 34:2, 141-164.

Tur-Viñes, Victoria; Fonseca-Mora, $\mathrm{M}^{\mathrm{a}}$ Carmen; Gutiérrez San Miguel, Begoña (2012). Ética de la publicación científica: iniciativas y recomendaciones. // El profesional de la información. 21:5, 491-497.

Tur-Viñes, Victoria; Fonseca-Mora, $\mathrm{M}^{\mathrm{a}}$ Carmen; Gutiérrez San Miguel, Begoña (2013). Ética y conflictos de autoría en la publicación científica. La opinión del editor, en La colaboración científica: una aproximación multidisciplinar. Gregorio González Alcaide; Javier Gómez Ferri; Víctor Agulló Calatayud (coords.) Valencia: Ñau Llibres. 153-168.

Tur-Viñes, Victoria; López Sánchez, Carmen; García del Castillo, José Antonio; López Orneales, Maricela; Monstserrat Gauchi, J.; Quiles Soler, M. C. (2014). Especialización y revistas académicas españolas de Comunicació. // Revista Latina de Comunicación Social 69, 12-40.

Valero Matas, Jesús; Jiménez García, Elena; Coca, Juan R. (2013). La perspectiva socio-hermenéutica en la evaluación del trabajo científico en España, en Investigación bibliotecológica. Méjico: UNAM, Instituto de Investigaciones Bibliotecológicas y de la Información.

Wiley, John y Sons, Ltd. (2014). Best Practice Guidelines on Publishing Ethics. A Publisher's Perspective. Second Edition. WILEY / Best Practice Guidelines on Publishing Ethics. COPE.

Wager, Elizabeth; Fiack, Suzan; Graf, Chris; Robinson, Andrew; Rowlands, lan (2009). Science journal editors' views on publication ethics: results of an international survey. // Journal of Medical Ethics. 35:6.

Wilkes, Michael S.; Kravitz, Richard L. (1995). Policies, practices, and attitudes of North American medical journal editors. // Journal of general internal medicine.10:8, 443450 .

Yankauer, Alfred (1990). Editor's report: scientific misconduct and the responsibility of journal editors. // American journal of public health. 80:4, 399-400.

Enviado: 2016-03-16. Segunda versión: 2016-06-17 Aceptado: 2016-09-15. científicas españolas de Comunicación, Educación y Psicología. // Ibersid. 10:2 (2016) 53-60. ISSN 1888-0967. 